Mots. Les langages du politique

$96 \mid 2011$

Les discours politiques. Approches interactionnistes et multimodales

\title{
Individualisme. Du Producteur à Tocqueville
}

\section{Marie-France Piguet}

\section{(2) OpenEdition \\ Journals}

Édition électronique

URL : https://journals.openedition.org/mots/20321

DOI : $10.4000 /$ mots. 20321

ISSN : 1960-6001

Éditeur

ENS Éditions

\section{Édition imprimée}

Date de publication : 5 septembre 2011

Pagination : 133-147

ISBN : 978-2-84788-316-9

ISSN : 0243-6450

Référence électronique

Marie-France Piguet, «Individualisme. Du Producteur à Tocqueville », Mots. Les langages du politique

[En ligne], 96 | 2011, mis en ligne le 05 septembre 2013, consulté le 22 avril 2022. URL : http://

journals.openedition.org/mots/20321; DOI : https://doi.org/10.4000/mots.20321 


\section{Individualisme. Du Producteur à Tocqueville}

Mot des sciences sociales contemporaines, de la modernité politique et du langage courant, individualisme concentre, à travers ses divers emplois, nombre de questions sur le rapport qu'entretient l'individu avec lui-même et avec les autres dans le monde moderne. Pour cette raison peut-être, sa signification incertaine a été maintes fois soulignée. Dès la première édition de son Vocabulaire technique et critique de la philosophie, André Lalande notait par exemple: «Mauvais terme, très équivoque, dont l'emploi donne lieu à des sophismes continuels ${ }^{1}$. Cette critique est restée présente tout au long des rééditions de l'ouvrage qui se sont succédé au cours du $20^{\mathrm{e}}$ siècle, et chez bien d'autres auteurs.

En amont de ces jugements, Tocqueville avait pris soin de le définir très longuement avant de l'employer en 1840 dans le deuxième volume de sa Démocratie en Amérique, après avoir mentionné que «l'individualisme est une expression récente qu'une idée nouvelle a fait naître ». À cette date, si individualisme reste un mot nouveau, ce n'est cependant plus un véritable néologisme : les dictionnaires l'ont enregistré dès 1834, et il possède déjà une histoire dont Tocqueville hérite et qu'il va poursuivre.

C'est le trajet qui a conduit le mot individualisme de ses premières attestations connues jusqu'à sa définition par Tocqueville, et plus largement jusqu'au milieu du $19^{\text {e }}$ siècle environ - le parcours de sa genèse en quelque sorte -, qui fait l'objet de cette étude, au sein d'une interrogation plus générale sur la difficulté de produire un énoncé définitoire à son sujet. Il sera guidé par le questionnement des rapports initiaux d'individualisme avec socialisme, égoïsme et, dans une moindre mesure, individualité, mots avec lesquels il se trouve très souvent associé à cette époque. Par ce parti pris d'investigation qui leste le mot de ses relations sémantiques passées, il s'agit pour nous d'éprouver la distance avec le contemporain et de travailler à reconstituer autant que faire se peut «l'étrangeté du temps passé » (Clavero, 1996, p. 195), pour mieux appréhender ce qui se joue par l'installation de ce signe lexical dans la langue.

1. Bulletin de la Société française de philosophie, t. IX, 1909, entrée «Individualisme », repris dans Lalande, 1926, p. 500. 


\section{Discours saint-simonien et mise en circulation du mot individualisme}

Selon le dictionnaire Trésor de la langue française, nous devons la première attestation connue du mot individualisme à un article de Pierre-Isidore Rouen publié dans le journal saint-simonien Le Producteur. Le TLF retient la date de 1825, mais il ne semble pas qu'individualisme soit attesté avant le numéro 17 du journal paru en janvier 1826. Les dictionnaires ont coutume de dater et de référencer avec précision l'apparition d'un nouvel élément lexical, mais la première occurrence écrite d'un mot nouveau est une donnée des plus fragiles qui témoigne, au mieux, d'une création dont les premières manifestations nous resteront à jamais inconnues. Aussi, la poursuite de l'enquête en amont des références retenues par les ouvrages lexicographiques se révèle souvent fructueuse pour une histoire des usages langagiers. On rencontre en effet dès 1815 le mot individualisme sous la graphie éphémère de individuel(I)isme. Par ailleurs, en 1823, une société regroupant d'anciens membres de la Charbonnerie a eu pour nom «société d'Individualistes "², et un extrait du Journal ecclésiastique de Rome publié en 1825 dans le Mémorial catholique a pour titre : «De l'individualisme, considéré par rapport à la religion et à la morale »3.

Les attestations du Producteur (1825-1826) enregistrées par la lexicographie contemporaine ne constituent donc qu'un relais dans la diffusion du néologisme, mais un relais essentiel. Elles offrent en effet un usage que l'on pourrait dire presque socialisé du mot, car elles sont plurielles (une quinzaine environ) et énoncées par différents rédacteurs. De plus, selon notre enquête, la traduction de certains écrits saint-simoniens a eu une part importante dans la diffusion du mot en anglais et en allemand (Piguet, 2008, p. 40-42).

Sans trop nous attarder sur ces occurrences, on peut rappeler que le mot individualisme est employé dans Le Producteur pour désigner de manière négative la pensée de la liberté individuelle et de l'autonomie intellectuelle. Ce sont d'abord les idées de Charles Dunoyer et de Benjamin Constant qui ont été visées par ce mot nouveau, celles qui font référence à la « liberté de conscience» promue par la Réforme, celles de la philosophie des Lumières, puis plus largement au fil des numéros, les idées qui ont engendré la dissolution des liens sociaux consécutive à la Révolution. Dans un contexte discursif qui défend la nécessité d'une réorganisation sociale, le néologisme individua-

2. Pour le détail de ces attestations dispersées et leurs références, je me permets de renvoyer à mes précédentes études : Piguet, 2005 et 2008 . Certaines analyses de cette partie de l'article abrègent en effet la question, exposée dans ces études, du contexte langagier et politique de l'émergence du mot.

3. Le Mémorial catholique, t. IV, Paris, Lachevardière fils, 1825, p.48-49. Tous mes remerciements à Lucien Jaume pour m'avoir signalé cette attestation. 
lisme exprime, pour en stigmatiser les effets, une conception liée au passé, qui accorde la première place à la liberté individuelle dans un système politique.

Les premières attestations du mot dans Le Producteur appartiennent au compte rendu critique d'un ouvrage de Charles Dunoyer, L'Industrie et la Morale considérées dans leur rapport avec la liberté, publié à la fin du mois de janvier 1826 par Pierre-Isidore Rouen. L’article porte «sur les idées générales ou les principes du système de $M$. Dunoyer [...]. L'idée la plus générale de ce système est la conception de l'homme individuel, de ses besoins et de ses facultés, comme bases uniques de la politique, et principes générateurs des lois sociales» (p.158-159):

Beaucoup de partisans de la liberté individuelle, non contens de la poser comme droit, la prescrivent comme devoir; ils vont jusqu'à imposer à chacun de ne penser et de n'agir que d'après lui-même, et protesteraient presque contre la domination bienfaisante qu'exercent infailliblement les hommes éclairés sur toutes les classes de la société. Ce sont ces exagérations de l'idée de liberté individuelle que nous repoussons; [...] Cependant l'individualisme comme base positive de la morale privée a, par cela même, une valeur critique par rapport à la politique; c'est-à-dire qu'il est le plus puissant adversaire de tous les systèmes vicieux, bien qu'il n'ait point la vertu d'en engendrer un lui-même. Nous lui devons la destruction de l'ancien ordre social. [...] L'individualisme depuis son origine, a cependant été sans cesse offert à la société comme système politique; et dans ces derniers temps, M. Dunoyer vient le reproduire sous de nouvelles formes. 4

Dunoyer n'employant pas le mot individualisme, il s'agit donc d'une reformulation de son «système » par ce mot qui fait alors écho, sous une forme condensée, à l'expression «conception de l'homme individuel»; il en subsume les différents traits qui sont clairement exprimés : «l'homme individuel», «les droits individuels », «la souveraineté individuelle», «la liberté individuelle». De ces traits, individualisme privilégie celui de «liberté individuelle» et met l'accent sur les «exagérations de l'idée de liberté individuelle» énoncées en amont : ne penser et n'agir que d'après soi-même et protester contre la domination des hommes éclairés.

Individualisme désigne, dans cet énoncé, la philosophie critique du 18 e siècle qui a conduit à la Révolution, et la théorie de Dunoyer comme «système politique» issu de cette philosophie. Il exprime en même temps ce que Rouen discrédite dans la théorie de Dunoyer, un système qui ne voit dans la société "autre chose qu'une collection d'hommes» (p.160) et qui fait de la liberté «le but de la société» (p.165). Dans les écrits saint-simoniens, individualisme est un mot qui dénonce, un mot connoté de manière négative car il exprime une résistance à «l'organisation sociale» dans un contexte qui la promeut.

4. Le Producteur, janvier 1826, nº 17, t. II, p. 162-163. 
Les numéros suivants du Producteur attestent également quelques occurrences du mot où s'affirme un infléchissement vers la dénotation d'un comportement social. C'est ainsi que sont fustigées «les excitations d'un individualisme barbare et la puissance illimitée du plus fort, dans l'état de nature »5. Ce déplacement métonymique de la désignation d'une doctrine à celle du comportement, de l'attitude qui lui est associée, se maintiendra dans la langue, le $T L F$, par exemple, organisant son entrée individualisme autour de cette grande distinction. Nous verrons Tocqueville privilégier ce dernier aspect de la désignation du mot.

\section{Association/individualisme/socialisme}

Les attestations du mot individualisme dans Le Producteur sont dues aux rédacteurs les plus influents au sein du journal, Rouen, Laurent et Enfantin, ceux qui feront partie du groupe initial des saint-simoniens réuni durant l'année 1829 pour professer un enseignement régulier des principes de l'École qui sera publié sous le titre Doctrine de Saint-Simon. Dans cet ouvrage collectif qui plaide pour une organisation sociale centrée sur la production industrielle et l'établissement d'un pouvoir spirituel fondé sur une connaissance positive de l'homme, individualisme entre dans le paradigme des termes qui définissent «la série critique»:

Oui, mon ami, ces mots, ordre, religion, association, dévoûment [en italique dans le texte], sont une suite d'hypothèses correspondantes à celles-ci : désordre, athéisme, individualisme, égoïsme. Tu trouveras peut-être que je traite bien mal la série organique, en lui donnant le même fondement qu'à la série critique, en les rattachant l'une et l'autre à deux conjectures. ${ }^{6}$

Il participe ainsi à l'énoncé du dogme de la pensée saint-simonienne, dans un antagonisme avec le mot association qui contribue à l'introduire dans le discours des réformateurs sociaux. Aussi, d'emblée, il se trouve opposé à société mais surtout à socialisme. L'invention même du mot socialisme, si l'on en croit Pierre Leroux, a résidé dans la nécessité de créer un antonyme à individualisme, et serait à mettre à son crédit : «C'est moi [...] qui, le premier, me suis servi du mot socialisme. Je forgeai ce mot par opposition à individualisme, qui commençait à avoir cours. Il y a de cela environ vingt-cinq ans. ॥7

En fait, Pierre Leroux comptera seulement parmi les premiers utilisateurs du mot socialisme 8 , mais on lui doit une des premières manifestations de l'op-

5. Le Producteur, août 1826, deuxième cahier, t. IV, p. 276-277.

6. Voir Doctrine de Saint-Simon, 1924, p. 464-465.

7. Voir Gans (1969, p. 45-58) qui cite cet énoncé de Pierre Leroux daté de 1857.

8. Selon Halévy, "Supplément» au Vocabulaire technique et critique de la philosophie, Paris, PUF, 1988 (16e édition), p. 1276. Sur cette question, voir les indications fournies par Dubois, 1962, p. 421. 
position entre les deux nouveaux termes dans un article publié dans la Revue encyclopédique en 1833, "Philosophie sociale ${ }^{9}$ :

Les partisans de l'individualisme se réjouissent ou se consolent sur les ruines de la société, réfugiés qu'ils sont dans leur égoïsme, les partisans du socialisme, marchant bravement à ce qu'ils nomment une époque organique, s'évertuent à trouver comment ils enterreront toute liberté, toute spontanéité sous ce qu'ils nomment l'organisation. (p.376)

Individualisme est rapporté à l'économie politique anglaise «qui, au nom de la liberté, fait des hommes entre eux des loups rapaces, et réduit la société en atomes» (ibid., p.374), à la maxime «chacun chez soi, chacun pour soi », à l'abandon de toute "providence sociale» pour les plus faibles. Socialisme fait référence aux théories saint-simoniennes qui «parlent de nous organiser en régiments de savants et en régiments d'industriels » et «s'avancent jusqu'à déclarer mauvaise la liberté de pensée» (p.377).

L'antonymie discursive entre individualisme et association/socialisme ainsi lancée s'installe durant la première moitié du $19^{\mathrm{e}}$ siècle et ensuite, principalement dans les écrits des diverses mouvances utopiques et socialistes. Au sein de ces discours politiques, elle est considérée comme allant de soi et participe d'une rhétorique souvent manichéenne à la manière de Cabet, qui soutient que «[les prolétaires] remueront la Société jusqu'à ce que le Socialisme ait remplacé l'odieux individualisme ${ }^{10}$. Toutefois, au-delà des énoncés militants, elle constitue une matrice capable d'intégrer des paramètres variés, et elle s'énonce alors de manière plus indirecte, plus diffuse mais plus argumentée aussi. C'est le cas des Lettres sur l'Amérique du Nord du saintsimonien Michel Chevalier, dont la traduction en allemand (1837) et en anglais (1839) a contribué à l'établissement des mots individualism et individualismus dans ces langues. Dans ce recueil, le mot individualisme désigne une propriété caractéristique de la vie sociale et politique des États de l'Amérique du Nord, que Chevalier rapporte au protestantisme et à la forme politique qui lui est liée, "le républicanisme ${ }^{11}$. Chevalier construit une opposition très structurante entre «l'Yankee, seul avec sa femme au milieu des bois», et «le Français [...] éminemment social» (p.72), qui incarnent deux principes différents, voire antagonistes, d'organisation sociale : le «principe d'autorité, c'est-à-dire d'association hiérarchique» pour la France, celui «d'indépendance, d'individualisme, de concurrence » pour l’Amérique du Nord. En France, nous agissons «principalement sous l'invocation des principes d'association et d'unité, qui

9. Revue encyclopédique, octobre-décembre 1833, t. LX, p.94-116. Je cite d'après Leroux, 1978, p. $365-380$.

10. Cabet, 1842, cité par Dubois, 1962, p. 125.

11. Les Lettres surl'Amérique du Nord, initialement publiées dans le Journal des Débats, ont connu plusieurs éditions revues, corrigées, augmentées. Je cite ici le t. Il de la quatrième édition. Les énoncés cités appartiennent à des lettres datées du 20 juillet et du 3 septembre 1835. 
sont caractéristiques du catholicisme et de la monarchie», et «nous ne nous sentons vivre que lorsque nous faisons partie d'un tout» (ibid., p. 169), mais il en va autrement en Amérique du Nord :

Sous l'influence du protestantisme et de la république, le progrès social s'est opéré par le procédé du morcellement poussé à sa limite extrême, l'individualisme : car protestantisme, républicanisme et morcellement, c'est tout un. Les individus se sont déliés les uns des autres; chacun a isolé sa personnalité pour la renforcer; ou si l'on s'est associé, l'on n'a constitué que des associations restreintes, sans aucun lien entre elles. (p. 168)

Plus généralement, l'antinomie entre les deux mots cristallise l'opposition de deux systèmes de pensée. Dans un ouvrage qui explore les solutions sociales à apporter pour soulager la misère après la révolution de 1848, Armand de Melun utilise les deux mots pour désigner les doctrines adverses qui ont prévalu au cours des événements. Le chapitre 2 de l'ouvrage a pour titre «Du socialisme», tandis que le chapitre 3 s'intitule «De l'individualisme». Pour l'auteur, le socialisme nie la liberté, et à «l'opposé du socialisme », il définit l'individualisme, lié ici aussi à l'économie politique, à la maxime «laisser faire, laisser passer», à Malthus tout particulièrement, comme

une doctrine [...] qui défend la liberté avec le même acharnement que l'attaque le socialisme, attribue autant de bien à la concurrence que celui-ci lui reproche de mal et rend à l'individu tout ce que son antagoniste veut donner à l'État. [...] Si le socialisme exagère le pouvoir et le devoir de la société, l'individualisme méconnaît sa mission et son but. (Melun, 1849, p. 15-18)

Très important pour sa diffusion, cet antagonisme initial qui entérine la désignation par ce mot d'une doctrine, d'un système de pensée et contribue à lui imprimer une connotation négative, sera enregistré lors de sa première définition lexicographique en 1834 par le Dictionnaire universel de la langue française, avec le latin de Pierre-Claude-Victoire Boiste (8e édition) : "Système d'isolement dans les travaux, les efforts; l'opposé de l'esprit d'association », et cette définition irriguera une très large partie des dictionnaires du $19^{\text {e }}$ siècle.

\section{Égoïsme / individualisme}

À la différence de cette relation d'opposition mise en œuvre tout particulièrement dans certains types de discours, celle de la proximité du mot individualisme avec celui d'égoïsme ne caractérise aucun registre particulier de langage. Il s'agit d'un voisinage sémantique régulièrement rappelé pour expliciter le dernier arrivé dans la langue, d'un hyperonyme souvent sollicité comme terme définitoire. On rencontre cette hyperonymie dès la première attestation connue du mot individualisme sous la graphie individuellisme : 
Un seul principe existait en France, l'individuellisme, l'universel égoïsme, fruit naturel d'un temps qui avait brisé tous les liens. Plus d'amour du prochain là où il n'y avait plus de religion pour en faire un précepte. Plus d'esprit de famille là où la famille se composait à peine du père et des enfants. Plus d'esprit de corps là où tout corps avait cessé d'exister. (Fauveau de Frénilly, 1815, p. 27)

D'une façon plus intéressante pour notre propos, car nous pouvons y voir la première définition d'un usage qui lie entre elles différentes dimensions de la vie sociale, l'article publié en 1825 par Le Mémorial catholique déploie les divers aspects référentiels de cette relation sémantique :

Je regrette que ce mot individualisme [en italiques dans le texte] sente le néologisme, mais il exprime parfaitement la situation morale de la société. Le mot isolement ne présente plus la même idée. Un homme peut s'isoler sans rapporter tout à lui-même; l'égoïsme, que l'on me pardonne l'expression, centralise les affections; l'individualisme a un degré de plus, il rapporte au seul moi humain non seulement les affections, mais les pensées, les habitudes et la croyance. (p.48)

Égoïsme est défini par le Dictionnaire de l'Académie française (1835) comme «vice de l’homme qui rapporte tout à soi ». Il désigne en même temps, par glissement métonymique, l'attitude, la conduite de celui que ce vice habite. À la différence de individualité, mot du vocabulaire philosophique à valeur neutre ou positive avec lequel celui d'individualisme partage une plage sémantique commune au moment de son émergence en raison de leur commune dérivation d'un même adjectifi2, celui d'égoïsme appartient au vocabulaire de la morale où il est affecté d'une valeur toujours négative, dont témoigne le choix du terme «vice» par l'Académie pour le définir. Toutefois, depuis la fin du $18^{\mathrm{e}}$ siècle au moins, le mot a échappé à ce seul registre et étendu son usage à celui du politique. Il s'agit moins d'une modification sémantique que d'un déplacement de la sphère de son emploi, qui a pour effet de mettre l'accent sur les conséquences de ce vice dans la vie politique.

L'un des exemples les plus significatifs pour cette étude est celui du maître à penser des saint-simoniens, le comte de Saint-Simon, décédé peu avant la publication du Producteur. Saint-Simon n'emploie pas le mot individualisme, comme l'ont déjà souligné Célestin Bouglé et Élie Halévy, mais il reprend à son compte cette extension de l'usage du mot égoïsme : "La décadence des doctrines générales anciennes a laissé développer l'égoïsme, qui envahit de jour en jour la société » (Saint-Simon, 1977, p. 21). Il voit dans ce vice et ses conséquences sociales le résultat et le caractère d'une époque historique précise et donne en quelque sorte une dimension historique au mot égoïsme, qui désigne alors une «maladie politique », celle qui conduit la société à sa « dissolution » (ibid., p. 104).

12. Sur cette proximité et l'examen de ses attestations, voir Piguet, 2005, p.116-122. 
Durant la première moitié du $19^{\mathrm{e}}$ siècle, nombre d'emplois du mot égoïsme, dans les discours sur la société, pourraient être reformulés rétrospectivement par celui d'individualisme. De même que individualité a pu exprimer un temps, dans certains écrits, ce que plus tard on nommera individualisme, il semble que égoïsme ait aussi tenu ce rôle, mais avec des valeurs bien différentes.

Ce phénomène est particulièrement sensible dans la presse ouvrière qui rend compte, sous la monarchie de Juillet, des interrogations nées du développement industriel et des premières révoltes ouvrières. On peut prendre pour exemple le journal lyonnais L'Écho de la Fabrique (1831-1834), journal des «chefs d'ateliers et des ouvriers en soie » qui fait connaître les revendications des canuts lors des révoltes de 1831 et de 1834 . Le mot individualisme y est attesté deux fois seulement, et par des articles tirés d'autres journaux ${ }^{13}$, alors que celui d'égoïsme est régulièrement employé par les contributeurs de L'Écho. Ces derniers dénoncent chez les «commerçants» ou «fabriquants», avec lesquels ils sont en conflit pour l'application du «tarif» négocié, un comportement social inspiré par le sentiment de «l'égoïsme». Ainsi pour l'auteur, resté anonyme, d'un article qui cherche à expliquer les causes profondes de "la décadence de la fabrique d'étoffes de soie», «ce n'est point dans l'augmentation du nombre d'ouvriers, ni dans la concurrence qu'on doit voir la décadence de la soierie. Le mal est dans l'égoïsme de quelques commerçans; eux seuls ont fait tomber cette industrie dans l'état où elle est » ${ }^{14}$.

D'une manière plus décisive à nos yeux, car l'application du mot s'étend alors à l'ensemble du «corps social » et peut être rapporté aux «institutions sociales ", L'Écho de la Fabrique emprunte à un journal stéphanois, Le Vulcain, un article explicitement consacré à la thématique générale de l'égoïsme, qui affiche pour titre «De l'égoïsme» et débute en ces termes :

L'égoïsme est la plaie la plus profonde du corps social, et il ne tend à rien moins qu'à le frapper de mort. [...] Il isole l'homme dans lui-même ; il tend à étendre la prédominance absolue de moi. [...] Oui, c'est à l'égoïsme qu'il faut imputer nos divisions et nos misères, mais c'est peut-être au vice de nos institutions sociales qu'il faut imputer l'égoïsme. ${ }^{15}$

La proximité entre individualisme et égoïsme a souvent été rappelée dans les courants utopiques et socialistes du $19^{\mathrm{e}}$ siècle, avec pour objectif la stigmatisation de celui d'individualisme. Mais, et c'est probablement moins connu, elle a aussi été discutée très tôt dans ce même registre, au motif qu'elle renvoie à une définition partielle du mot individualisme et donne des arguments faciles aux

13. Dans Le Sémaphore de Marseille, 1832, et dans Le Journal progressif de l'Aube, 1833. L'Écho de la Fabrique est désormais consultable en version image et en version numérisée [http://echofabrique.ens-lyon.fr/]. Site consulté le 25 janvier 2011.

14. «De Lyon et de la concurrence étrangère », L'Écho de la Fabrique, 6 novembre 1831, p. 2.

15. Le Vulcain, cité par L'Écho de la Fabrique, 19 août 1832, p. 4. 
ennemis de la liberté individuelle. C'est ainsi que le journal de l'École sociétaire, La Phalange, accusé par les saint-simoniens de défendre l'égoïsme, est conduit à préciser que « le mot individualisme [en italiques dans le texte] peut se prendre en effet en bonne ou mauvaise part; dans son sens défavorable, et c'est dans ce sens évidemment que le prennent nos critiques, leur reproche revient à ceci : votre système est la théorie de l'égoïsme » ${ }^{16}$. Mais ce sens " antisocial» ne met en lumière «qu'une des deux faces de la chose» (p. 95), alors qu'«il est dans la langue un autre sens du mot individualisme, auquel les fouriéristes n'ont aucune raison de répugner comme expression, un peu vague [...] mais, après tout, fidèle de leur idéal». L'auteur, Delmas, définit alors comme suit le sens du mot individualisme dont se réclame, selon lui, l'École sociétaire:

Pris dans son sens favorable, qu'est-ce donc que l'individualisme? Pour la sphère de la pensée, il n'est autre que l'aperception réfléchie que chacun a de soi comme personnalité libre; pour la sphère du sentiment, il est la conscience spontanée, instinctive, que nous avons tous de nos besoins, de notre valeur, de nos vocations diverses; pour la sphère des faits, il est la réalisation de lui-même dans toutes les fonctions de la vie. Rien n'est donc plus légitime en soi que l'individualisme. (Ibid., p. 88-89)

En tirant la définition du mot individualisme du côté de la dénotation de celui d'individualité, tout en forçant le trait de la critique qui lui est adressée par l'emploi de celui d'égoïsme, l’École sociétaire explicite à sa manière les tensions axiologiques qui traversent l'usage d'individualisme et montre en même temps que ces tensions ont été précocement ressenties, explicitées et... exploitées.

Il apparaît que dès ses débuts, individualisme est déchiré entre une valeur philosophique positive qui défend le sentiment intime et l'épanouissement de soi en faisant référence à la notion d'individualité, et une valeur morale condamnant un amour de soi qui conduit à une indifférence envers autrui. L'ambivalence ainsi mise en lumière ne manque pas d'actualité et l'on peut penser qu'elle n'est pas sans conséquences dans les difficultés toujours rencontrées pour déchiffrer sa signification.

\section{Égoïsme/individualisme : Tocqueville}

Dans une toute autre perspective que celle de l'École sociétaire, mais selon une manière récurrente d'insérer le mot dans le discours, c'est en travaillant sa distance avec celui d'égoïsme que Tocqueville va marquer la spécificité du dernier attesté, dans son ouvrage De la Démocratie en Amérique.

De la Démocratie en Amérique se compose de deuxvolumes: le premier a été publié en 1835 , le second en 1840 . Tocqueville n'emploie individualisme qu'à partir du second volume, précisément à partir du second chapitre de sa deuxième

16. La Phalange, «Individualisme et solidarité », 1848 [1836], t. II, vol. VII, p. 88. 
partie consacrée à l' "Influence de la démocratie sur les sentiments des Américains ». À cette date, le mot individualisme n'est plus un néologisme. Enregistré par les dictionnaires, il appartient à la longue liste des nouveaux termes qui se sont répandus au sein du langage parlementaire aux alentours de 1830 (Matoré, 1967, p. 28 et 41), et il a débuté sa carrière dans la littérature, chez Balzac en particulier ${ }^{17}$, mais aussi chez Lamartine, notant que «la civilisation, en avançant, réduit la pensée religieuse à l’individualisme » (Lamartine, [1835] 1861, p. 277). Toutefois, il reste encore peu employé, appartient principalement au vocabulaire des publicistes et sa portée se limite souvent au cadre de la polémique. Il n'en va pas tout à fait de même avec Tocqueville, et c'est peut-être la raison pour laquelle on a longtemps pensé que la traduction de Démocratie en Amérique était à l'origine du mot dans la langue anglaise (Claeys, 1986, p. 81-86).

Individualisme est introduit parune longue définition de laquelle dépendent tous ses emplois. On en compte treize occurrences ${ }^{18}$, dont six dans ce contexte définitoire et quatre dans le titre des chapitres. Tocqueville affiche donc le mot individualisme plus qu'il ne l'emploie réellement dans le cours de son texte, et il manifeste dans son usage à la fois une grande rigueur et une forte originalité en procédant d'abord par sa définition'19. Il semble l'employer de manière très prudente.

Avec près de la moitié de ses occurrences consacrées à sa propre définition, et même s'il n'y a pas pour Tocqueville «de bonne langue sans termes clairs» (Tocqueville, 1981, p. 86), on peut s'interroger sur un tel souci définitoire en dehors de tout contexte polémique, et cela d'autant plus qu'il s'agit là d'un retour réflexif sur un terme qui appartient à son vocabulaire depuis 1835 au moins, date à laquelle il l'emploie dans ses Carnets de voyages, puis dans d'autres textes restés manuscrits ${ }^{20}$. En qualifiant de « récente » cette « expression », l'auteur donne à penser qu'il a voulu éclaircir une nouveauté langagière encore mal établie. Il s'agit bien sûr d'un mot nouveau, mais de plus, c'est un mot abstrait. Or ces deux qualités sont propres à une évolution du langage que Tocqueville juge sévèrement. Dans un chapitre consacré aux modifications apportées par la démocratie américaine à la langue anglaise et, plus généralement, aux évolutions langagières induites par la démocratie, véritable chapitre de sociologie du langage avant la lettre, il a identifié et analysé les différentes caractéristiques des «langues démocratiques » parmi lesquelles, précisément, la création de mots nouveaux et la fréquence d'emploi des mots abstraits qui «rendent l'expression plus rapide et l'idée moins nette» (ibid., p. 89). Le mot

17. Dans Le Médecin de campagne (1833), cité par Dubois, 1962, p. 322.

18. Données Frantext. L'Ancien Régime et la Révolution compte quatre occurrences du terme.

19. Sur la définition par Tocqueville des notions capitales mises en œuvre dans son ouvrage, voir Guellec, 2004, p. 241.

20. Eduardo Nolla en cite quelques exemples dans son introduction à la date de 1835 (Tocqueville, 1990, p. xlix). 
individualisme appartenant à ces deux catégories critiquées, il s'agit pour l'auteur d'expliciter ce nouvel élément de la langue avant d'en faire usage.

Il n'est pas certain cependant que cette raison soit suffisante ${ }^{21}$. Tocqueville en effet ne définit pas le mot d'une manière abstraite, comme les dictionnaires par exemple, mais au sein de sa propre pensée, en précisant par touches successives en quoi cette « idée nouvelle » qu'énonce "une expression récente», se rattache à - et se détache de - celle d'égoïsme. En procédant de la sorte, il l'explicite pour exprimer une notion conçue par lui dans son analyse de la société américaine et plus largement celle des «peuples démocratiques».

La définition ouvre le deuxième chapitre de la deuxième partie du volume et son titre, «De l'individualisme dans les pays démocratiques», offre donc la première occurrence du mot.

Au cours du chapitre précédent, Tocqueville a montré pourquoi « les peuples démocratiques» sont moins attachés à la liberté qu'à l'égalité telle que l'on peut l'observer en Amérique et plus largement dans les nations modernes : absence de différence héréditaire de conditions, droit pour tous « de se livrer aux mêmes plaisirs, d'entrer dans les mêmes professions, de se rencontrer dans les mêmes lieux; en un mot de vivre de la même manière et de poursuivre la richesse par les mêmes moyens " (p.119-120). Il a aussi noté que seuls « les gens attentifs et clairvoyants [...] aperçoivent les périls dont l'égalité nous menace » (p.121), mais sans expliciter alors la nature des périls évoqués, précisant seulement que «les maux que l'extrême égalité peut produire ne se manifestent que peu à peu; [qu']ils s'insinuent graduellement dans le corps social» (p.121). Ce n'est qu'ensuite, dans les chapitres suivants, qu'il montre en quoi l'« individualisme» constitue ce danger, et comment il peut être combattu.

Aussi, la définition du mot individualisme en ouverture du deuxième chapitre, associée à sa première occurrence dans le titre même du chapitre, prendelle un relief très particulier :

J'ai fait voir comment, dans les siècles d'égalité, chaque homme cherchait en luimême ses croyances; je veux montrer comment, dans les mêmes siècles, il tourne tous ses sentiments vers lui seul.

L'individualisme [en italiques dans le texte] est une expression récente qu'une idée nouvelle a fait naître. Nos pères ne connaissaient que l'égoïsme.

L'égoïsme est un amour passionné et exagéré de soi-même, qui porte l'homme à ne rien rapporter qu'à lui seul et à se préférer à tout.

L'individualisme est un sentiment réfléchi et paisible qui dispose chaque citoyen à s'isoler de la masse de ses semblables et à se retirer à l'écart avec sa famille et ses amis; de telle sorte que, après s'être ainsi créé une petite société à son usage, il abandonne volontiers la grande société à elle-même.

21. Dans un brouillon, il écrit en effet à propos du mot individualisme : «Je ne crains point de me servir de mots nouveaux quand ils sont nécessaires pour peindre une chose nouvelle.» (Tocqueville, 1990, p. 97) 
L'égoïsme naît d'un instinct aveugle; l'individualisme procède d'un jugement erroné plutôt que d'un sentiment dépravé. II prend sa source dans les défauts de l'esprit autant que dans les vices du cœur.

L'égoïsme dessèche le germe de toutes les vertus, l'individualisme ne tarit d'abord que la source des vertus publiques; mais à la longue, il attaque et détruit toutes les autres et va enfin s'absorber dans l'égoïsme.

L'égoïsme est un vice aussi ancien que le monde. Il n'appartient guère plus à une forme de société qu'à une autre. L'individualisme est d'origine démocratique, et il menace de se développer à mesure que les conditions s'égalisent. (p.125)

Dans cette longue définition, entre le mot dont il souligne la nouveauté et le phénomène par lui exprimé, Tocqueville place le signifié de la tradition saussurienne, la conceptualisation du référent. Il est important de noter que l'«idée » est qualifiée de «nouvelle» et qu'en revanche son référent reste dans une indétermination temporelle. Sa description, donnée ensuite, laisse penser que sans se perdre dans la nuit des temps, il ne présente pas le même caractère d'extrême nouveauté, qu'il s'agit d'un processus dont la perception, la conscience que l'on peut en avoir, est récente. C'est d'ailleurs, semble-t-il, le sens de la mise en garde de l'auteur au cours du chapitre précédent.

Sa définition référentielle de l'individualisme est construite ensuite, dans un décalage mesuré avec égoïsme, en trois points principaux : la nature de ces phénomènes qui sont définis comme deux affects ramenant l'individu à lui-même et à ses proches; les effets destructeurs de ces affects dans la vie sociale; l'origine différente de ces affects. II met en lumière de la sorte ce qui distingue essentiellement pour lui l'individualisme de l'égoïsme, une valeur de jugement ${ }^{22}$ et une dimension sociale, politique, historique, rapportant, comme il a été souligné par les commentateurs, «l'égoïsme à l'homme, et l'individualisme au citoyen " $^{23}$. L'explicitation de la distance entre les deux mots, telle qu'il la donne à percevoir, permet de préciser que la nouveauté énoncée par le dernier attesté est corrélée au devenir démocratique des sociétés : "Non seulement la démocratie fait oublier à chaque homme ses aïeux, mais elle lui cache ses descendants et le sépare de ses contemporains; elle le ramène sans cesse vers lui seul et menace de le renfermer enfin tout entier dans la solitude de son propre cœur.» (p.127)

Dans ce qu'il nomme individualisme, il esquisse les traits d'une disposition intrinsèque aux hommes des sociétés démocratiques, dans laquelle il voit la conséquence de l'égalité des conditions. Parce qu'elle efface les rapports hiérarchiques et générationnels inhérents aux sociétés traditionnelles, l'égalité " place les hommes à côté les uns des autres, sans lien commun qui les retienne [et] les dispose à ne point songer à leurs semblables» (p. 131). Ce

22. Il note dans un brouillon : «L'égoïsme, vice du cœur; l’individualisme, de l'esprit». Cité par Nolla dans Tocqueville, 1990, p. 9.

23. Selon Lamberti, 1970, p.12. 
«sentiment» dont la composante intellectuelle, développée dans la première partie du volume, est rappelée au début de la définition : «[j’]ai fait voir comment, dans les siècles d'égalité, chaque homme cherchait en lui-même ses croyances», œuvre à une indifférenciation sociale qui enferme les individus en eux-mêmes. À cet égard, Tocqueville reprend, au profit de l'individualisme, la métaphore médicale maintes fois associée à l'égoïsme ; il s'agit aussi d'une «maladie», mais d'une maladie «naturelle au corps social dans les temps démocratiques» (p.132), dont la liberté serait le seul «remède efficace»: "Les Américains ont combattu par la liberté l'individualisme que l'égalité faisait naître, et ils l'ont vaincu.» (p.132) Concrètement, les Américains ont lutté contre l'individualisme par la pratique des associations en particulier, mais Tocqueville n'établit pas une opposition rhétorique et axiologique entre les mots individualisme et association, comme on a pu l'observer. Il exprime par ces deux mots le problème majeur auquel est confrontée, selon lui, la société démocratique et son possible dépassement.

Dans la pensée de Tocqueville, le mot individualisme désigne donc moins une conception ou une théorie qu'une attitude, un état d'esprit, un comportement, selon d'autres usages également attestés, mais beaucoup plus dispersés. Il énonce une manière d'être, propre aux individus qui appartiennent aux sociétés marquées par l'effacement des liens sociaux accordés de l'extérieur de chacun en quelque sorte, et sans le soutien desquels les hommes manifestent spontanément un repli sur eux-mêmes :

Les hommes qui habitent les pays démocratiques n'ayant ni supérieurs, ni inférieurs, ni associés habituels et nécessaires, se replient volontiers sur eux-mêmes et se considèrent isolément. J'ai eu l'occasion de le montrer fort au long quand il s'est agi de l'individualisme. (p. 359)

En choisissant de le définir par rapport à celui d'égoïsme, Tocqueville privilégie sa face sombre sans cependant lui donner une valeur négative. Sous sa plume, le mot reste neutre, de l'ordre du constat, mais il désigne une menace, une menace pour la démocratie elle-même. Plus tard, tirant les conséquences majeures de cette proposition initiale et manifestant toujours une forte sensibilité au caractère récent du mot, il en donnera une définition plus dépouillée, en appui sur le mot « individu» :

Nos pères n'avaient pas le mot d'individualisme [en italiques dans le texte], que nous avons forgé pour notre usage, parce que, de leur temps, il n'y avait pas en effet d'individu qui n'appartînt à un groupe et qui pût se considérer absolument seul. (Tocqueville, 1967, p.176)

Tocqueville inscrit son usage du mot dans le déploiement de traits bien attestés, tant par la prise en charge de l'indépendance intellectuelle de l'individu que par la désignation d'un comportement et le rappel de sa proximité avec 
l'égoïsme. D'une certaine façon, il recueille ces traits, mais en les rapportant conjointement à la dynamique égalitaire, dans laquelle il voit le phénomène qui rend compte du bouleversement des sentiments et des conduites humaines, il leur donne une raison commune et les rend solidaires. Il établit ainsi un usage que l'on peut dire sociologique du mot qui désigne alors une notion qui sera au centre de nombreuses interrogations dans les sciences sociales de la seconde moitié du $20^{\mathrm{e}}$ siècle.

Au terme provisoire d'un trajet où il a commencé à se faire jour avec la stigmatisation d'une théorie politique centrée sur l'individu et ses droits dans le prolongement de la philosophie des Lumières, le mot individualisme enregistre et cristallise chez Tocqueville, subsume en quelque sorte, diverses facettes d'un "sentiment », d'une conduite sociale inhérente aux individus appartenant aux sociétés dans lesquelles le rapport d'égalité s'est progressivement substitué aux relations hiérarchiques traditionnelles.

Entre la désignation d'une doctrine, d'un système de pensée et celle d'un comportement, d'une manière d'être, tout un continuum d'usages du mot, partagés par des valeurs contradictoires et dispersés dans des contextes divers, ont pu être observés. Ces usages mis en circulation durant la Restauration ont en commun de traiter, sous des angles multiples, de la relation de l'individu à la société dans ses dimensions les plus variées (politique, philosophique, familiale, religieuse, morale...). Difficiles à définir au-delà de la cartographie de leur éparpillement, ils apparaissent rétrospectivement comme l'expression langagière nouvelle d'une conscience partagée de la transformation de ce rapport.

\section{Références}

Chevalier Michel, 1844 [1835], Lettres sur l'Amérique du Nord, $4^{\mathrm{e}}$ édition, Bruxelles, Wouters.

CLAEYS Gregory, 1986, «Individualism, socialism and social science. Further notes on a process of conceptual formation, 1800-1850 », Journal of the History of Ideas, vol.XLVII, $\mathrm{n}^{\circ} 1$, janvier-mars.

CLAVERo Bartolomé, 1996 [1991], La grâce du don. Anthropologie catholique de l'économie moderne, trad. de l'espagnol par J.-F. Schaub, préface de J. Le Goff, Paris, Albin Michel.

Doctrine de Saint-Simon. Exposition. Première année, 1829, 1924 [1830], introduction et notes de C. Bouglé et É. Halévy, Paris, Rivière.

Duвoıs Jean, 1962, Le vocabulaire politique et social en France de 1869 à 1872, Paris, Larousse.

FAuVEAU DE FrÉNIlly François-Auguste, 1815, Considérations sur une année de l'histoire de France, Paris, Chaumerot.

GANS Jean, 1969, «Socialiste, socialisme», Cahiers de lexicologie, vol.XIV. 
GUELLEC Laurence, 2004, Tocqueville et les langages de la démocratie, Paris, Champion. HALÉVY Élie, 1988 (16e édition), «Supplément» au Vocabulaire technique et critique de la philosophie, Paris, PUF.

LALANDE André, 1926, Vocabulaire technique et critique de la philosophie, Paris, Alcan. LAMARTINE Alphonse (de), 1861 [1835], Souvenirs, impressions, pensées et paysages pendant un voyage en Orient, œuvres complètes, t. VI, Paris, publié par l'auteur. LAMBERTI Jean-Claude, 1970, La notion d'individualisme chez Tocqueville, Paris, PUF. Leroux Pierre, 1978, "De l'individualisme et du socialisme», Fuvres, 1825-1850, Genève, Slatkine Reprints.

MATORÉ Georges, 1967 [1951], 2e édition, Le vocabulaire et la société sous LouisPhilippe, Slatkine Reprints, Genève.

Melun Armand (de), 1849, De l'intervention de la société pour prévenir et soulager la misère, Paris, Hachette.

PIguet Marie-France, 2005, "Benjamin Constant et la naissance du mot "individualisme" ", Annales Benjamin Constant, n² 29, Genève, Slatkine p.101-124.

- 2008, "Individualisme. Origine et réception initiale du mot», Fuvres et critiques, Revue internationale d'étude de la réception critique des œuvres littéraires de langue française, vol.XXXIII, nº 1, p. 39-60.

SaInt-SIMon Claude-Henri (de), 1977 [1821], Du Système industriel, dans F Fuvres de Claude-Henri de Saint-Simon, Genève, Slatkine Reprints, t. III.

TocqueVILLE Alexis (de), 1967 [1856], L’Ancien Régime et la Révolution, Paris, Gallimard.

- 1981, De la Démocratie en Amérique, t. I [1835] et t. II [1840], Paris, Garnier Flammarion.

- 1990, De la Démocratie en Amérique, t.I [1835] et t.II [1840], Première édition historico-critique revue et augmentée par E. Nolla, Paris, Vrin. 